\title{
Qiyusanlong decoction suppresses lung cancer in mice via Wnt// -catenin pathway
}

\author{
JIA-BING TONG ${ }^{1-3}$, XING-XING ZHANG ${ }^{1,3}$, XIN-HENG WANG ${ }^{2}$, SHI-JIE ZENG ${ }^{2}$, \\ DAN-YANG WANG ${ }^{2}$, ZHI-QIANG ZHANG ${ }^{2}$, JIAN HU ${ }^{2}$, CHEN YANG $^{1,3}$ and ZE-GENG LI ${ }^{2,3}$ \\ ${ }^{1}$ Department of Respiratory, The First Affiliated Hospital of Anhui University of Chinese Medicine, \\ Hefei, Anhui 230031; ${ }^{2}$ Key Laboratory of Xin An Medicine, Ministry of Education, Anhui University \\ of Chinese Medicine, Hefei, Anhui 230012; ${ }^{3}$ Institute of Chinese Medicine for Respiratory Disease, \\ Anhui Provincial Academy of Chinese Medical Sciences, Hefei, Anhui 230031, P.R. China
}

Received June 5, 2017; Accepted November 2, 2017

DOI: $10.3892 / \mathrm{mmr} .2018 .8478$

\begin{abstract}
Lung cancer is one of the most fatal cancers due to its high metastatic rate. Traditional Chinese medicine has been used in cancer patients for decades to improve quality of life and prolong survival time. The present study used a novel Qiyusanlong (QYSL) decoction composed of 10 kinds of Chinese medicine including astragalus membranaceus (Huangqi), polygonatumod oratum (yuzu), scolopendra (tianlong), pberetima (dilong), solanum nigrum (longkui), herbahedyotis (baihushecao), semen coicis (yiyiren), euphorbia helioscopia (zeqi), curcuma longa (eshu) and tendril-leaved fritillary bulb (chuanbei). The effects and function of the QYSL decoction remain to be elucidated. The present study established a mouse xenograft model using Lewis lung carcinoma cell injection and administered different doses of QYSL decoction to the mice. It was demonstrated that the chemotherapy drug Cisplatin (DDP) and QYSL decoction repressed lung tumor growth, and the inhibitory effect of DDP was more significant. Furthermore, QYSL decoction and DDP modulated the expression of regulatory proteins in the Wnt/ $\beta$-catenin pathway, including Wnt1, Wnt2, Wnt5a and glycogen synthase kinase $3 \beta$, detected by western blotting, and affected the signals of cluster of differentiation 44 variation 6 and Survivin in tumor tissues, examined via immunohistochemistry. The combination of QYSL decoction and DDP enhanced
\end{abstract}

Correspondence to: Dr Ze-Geng Li, Key Laboratory of Xin An Medicine, Ministry of Education, Anhui University of Chinese Medicine, 1 Qianjiang Road, New Station District, Hefei, Anhui 230012, P.R. China

E-mail: zegengli2016@sina.com

Abbreviations: QYSL, qiyusanlong; TCM, traditional Chinese medicine; LLC, Lewis lung carcinoma cells; GSK3 $\beta$, glycogen synthase kinase 3 $\beta$; Dvl, Dishevelled; SFRP, Secreted frizzled-related proteins; CD44v6, cluster of differentiation 44 variation 6

Key words: qiyusanlong decoction, lung cancer, Wnt/ $\beta$-catenin pathway the inhibitory effect. These data demonstrated that the QYSL decoction repressed lung tumor development via the Wnt/ $\beta$-catenin pathway. The therapeutic effect of QYSL decoction alone was milder compared with DDP, however the combination of QYSL decoction and chemotherapy exhibited an increased the rapeutic effect compared with the treatments administered alone. These findings revealed the function of QYSL decoction as a lung cancer treatment and provided insight for a novel lung cancer therapy.

\section{Introduction}

Lung cancer is the most frequently diagnosed cancer and the prominent reason for tumor-associated mortalities across the globe (1). Chemotherapy alone or combined with other therapies has an important role in the treatment of lung cancer (2), an example of this includes Cisplatin (DDP)-based chemotherapy (3). Although remarkable advances have been made in targeted therapy, the median survival time of patients with advanced disease is typically less than a year (4). As an initial treatment, the cytotoxicity of DDP is effective; however, cancer cells frequently develop resistance to DDP as a result of its frequent usage (5). Therefore, the development of novel therapeutic strategies or agents is important for improving the survival rate of lung cancer patients.

Traditional Chinese medicine (TCM) has contributed greatly to the prosperity of the Chinese population for several thousand years and to the development of traditional medicine worldwide (6). As an adjuvant therapy, TCM reduces the toxicity and adverse reactions induced by surgery, chemotherapy and radiotherapy, by acting synergistically (7). TCM has been widely used in the treatment of tumors, however is not the first-line therapeutic and is second to modern western medicine in terms of mechanism elucidation and evidence-based data. Further developments in the area of TCM may enhance novel therapeutic strategies for cancer in the future.

A novel Qiyusanlong (QYSL) decoction formula was established by Professor Han Mingxiang (The First Affiliated Hospital of Anhui University of Chinese Medicine, Hefei, China), a doctor of TCM according to the folk prescription of 
'Fu Zheng Xiao Ji' (8). The QYSL decoction was composed of 10 variants of Chinese medicine including astragalus membranaceus (Huangqi), polygonatum odoratum (yuzu), scolopendra (tianlong), pberetima (dilong), solanum nigrum (longkui), herbahedyotis (baihushecao), semen coicis (yiyiren), euphorbia helioscopia (zeqi), curcuma longa (eshu) and tendril-leaved fritillary bulb (chuanbei). The QYSL decoction has been used in clinical study for over 20 years in China, however, research regarding the specific function of the QYSL decoction as a cancer therapeutic, particularly in lung cancer, is limited. The author's previously demonstrated that the QYSL decoction suppresses the level of programmed cell death (PD)-1/PD-L1 in mice bearing Lewis lung carcinoma (LLC) (8). To further investigate the role of QYSL decoction on the progression of LLC, the present study employed QYSL decoction to treat a C57BL/6 mouse xenograft model using LLC cells. The present study investigated the effect of QYSL decoction at three doses on lung cancer in a mouse model, and its association with the $\mathrm{Wnt} / \beta$-catenin signaling pathway. The canonical Wnt/ $\beta$-catenin pathway has an essential role in different stages of tumor development, including cancer cell proliferation, migration, invasion, tumorigenesis and metastasis (9). Wnt/ $\beta$-catenin signaling is tightly regulated at multiple cellular levels (10). Dysregulation of the Wnt/ $\beta$-catenin pathway may be an important factor contributing to increased maintenance and proliferation signaling in various cancers (11), including lung cancer (12). Numerous regulatory proteins are involved in Wnt/ $\beta$-catenin pathway, including Axin $2, \beta$-catenin, glycogen synthase kinase $3 \beta$ (GSK3 $\beta$ ), c-Myc, cyclin D1, Dishevelled (Dvl), Secreted frizzled-related proteins 2 (SFRP 2) and cluster of differentiation 44 variation 6 (CD44v6) (13). The present study demonstrated that QYSL decoction and DDP regulated the expression of regulatory proteins in Wnt $/ \beta$-catenin, including Wnt1, Wnt2, Wnt5a, GSK3 $\beta$, and affected the signals of CD44v6 and Survivin in tumor tissues.

The results of the present study revealed that QYSL decoction exhibited an inhibitory effect on lung cancer growth via the $W n t / \beta$-catenin pathway, which verified the direct anti-tumor effect of QYSL decoction in cancer. These data demonstrated that the combination of QYSL decoction and DDP may represent a novel future approach in treatment for lung cancer.

\section{Materials and methods}

Reagents. TRIzol ${ }^{\circledR}$ buffer (catalog no. 87801), RevertAidTM first strand cDNA Synthesis Kit (catalog no. 00174486) and enhanced chemiluminescence (ECL) kit (catalog no. QE218149) were purchased from Thermo Fisher Scientific, Inc., (Waltham, MA, USA). Polyclonal anti-Wnt1 (catalog no. AA56134), anti-GSK3ß (catalog no. CN33151), anti-phosphorylated (p)-GSK3 $\beta$ (catalog no. CN33151), anti-SFRP-2 (catalog no. CC22141) antibodies were purchased from Bioworld Technology, Inc., (St Louis Park, MN, USA). Monoclonal anti-Wnt2 (catalog no. GR203181-1), anti-Wnt5a (catalog no. CC36131), anti- $\beta$-catenin (catalog no. GR177612-31), anti-Survivin (catalog no. GR209492-1) and polyclonal anti-Dvl-1 antibodies (catalog no. 109145) were obtained from Abcam (Cambridge, UK). Anti-CD44V6 antibody (catalog no. bs-20756R) was purchased from BIOSS
(Beijing, China). DAB kit (catalog no. K137726A) was obtained fromOrigene Technologies, Inc., (Beijing, China).

All crudes of QYSL decoction drugs and Cisplatin (DDP; catalog no. 411016CE) were purchased from dispensary of TCM and dispensary for western medicine in the First Affiliated Hospital of Anhui University of Chinese Medicine (Hefei, China). The decoction consisted of $30 \mathrm{~g}$ astragalus membranaceus (huangqi), $10 \mathrm{~g}$ polygonatum odoratum (yuzu), $6 \mathrm{~g}$ scolopendra subspinipes mutilans (tianlong), $6 \mathrm{~g}$ pberetima (dilong), $20 \mathrm{~g}$ solanum nigrum (longkui), $20 \mathrm{~g}$ herbahedyotis (baihushecao), $20 \mathrm{~g}$ semen coicis (yiyiren), $6 \mathrm{~g}$ euphorbia helioscopia (zeqi), $10 \mathrm{~g}$ curcuma longa (eshu) and $6 \mathrm{~g}$ tendril-leaved fritillary bulb (chuanbei). QYSL decoction was stored at $4^{\circ} \mathrm{C}$ at a concentration of $4.024 \mathrm{~g} / \mathrm{ml}$.

Animals. A total of 60 male SPF grade C57BL/6 mice (6-8 weeks old), weighing $20 \pm 2 \mathrm{~g}$, were purchased from Cavens Laboratory Animal Co., Ltd. (Changzhou, China; catalog no. SCXK 2011-0003). Mice were housed in air-filtered laminar flow cabinets with a 12-h light cycle and food and water ad libitum. All the animal care and experimental procedures followed were approved by the Institutional Animal Care and Use Committee of Anhui University of Chinese Medicine. Lewis lung carcinoma LLCs were obtained from Institute of Shanghai Academy of Life Sciences, Chinese Academy of Sciences (Shanghai, China), and maintained in Dulbecco's modified Eagle's medium (Thermo Fisher Scientific, Inc., Waltham, MA, USA) supplemented with $10 \%$ fetal bovine serum (Thermo Fisher Scientific, Inc.), $100 \mathrm{U} / \mathrm{ml}$ penicillin and $100 \mathrm{U} / \mathrm{ml}$ streptomycin (Origene Technologies, Inc.), and kept in an incubator at $37^{\circ} \mathrm{C}$, in a humidified atmosphere containing $5 \% \mathrm{CO}_{2}$.

Tumor xenografts in nude mice. LLCs were suspended in serum-free medium and a total of $1 \times 10^{5}(0.1 \mathrm{ml})$ LLCs per mouse were inoculated subcutaneously into the left dorsal flanks of nude mice. When tumors reached an average volume of approximately $3-5 \mathrm{~mm}^{3}$ (10 days following injection), the mice were considered as established with xenografts. The next day, mouse models were randomized into six groups ( $n=8$ per group): i) Control group (C): Mice received dosage of $0.2 \mathrm{ml} / 10 \mathrm{~g}$ physiological saline via intragastric administration for 21 days, and $0.4 \mathrm{ml}$ of physiological saline by intraperitoneal injection once a week. ii) Low dosage of QYSL decoction group (LQ): Mice underwent intragastric administration of $20.12 \mathrm{~g} / \mathrm{kg}$ QYSL decoction for 21 days, and $0.4 \mathrm{ml}$ physiological saline via intraperitoneal injection once a week. iii) Medium dosage of QYSL decoction group (MQ): Mice underwent intragastric administration of $40.24 \mathrm{~g} / \mathrm{kg}$ QYSL for 21 days, and $0.4 \mathrm{ml}$ physiological saline via intraperitoneal injection once a week. iv) High dosage of QYSL decoction group (HQ): Mice received intragastric administration with doses of $80.48 \mathrm{~g} / \mathrm{kg}$ QYSL for 21 days, and $0.4 \mathrm{ml}$ physiological saline via intraperitoneal injection once a week. v) DDP group (DDP): Mice received intragastric administration of $0.2 \mathrm{ml} / 10 \mathrm{~g}$ physiological saline for 30 days, and $0.4 \mathrm{ml}$ DDP via intraperitoneal injection once a week. vi) HQ+DDP group: Mice received intragastric administration of $80.48 \mathrm{~g} / \mathrm{kg}$ QYSL for 21 days, and $0.4 \mathrm{ml}$ DDP via intraperitoneal injection once a week. Following a 22-day period, tumor xenografts were 
isolated, and measured with the precision electronic balance and the tumor inhibition ratio was calculated. The tumor inhibition ratio=(the average quality of tumor in $\mathrm{C}$ group-the average quality of tumor in experience group)/the average quality of tumor in C group x $100 \%$.

Reverse transcription-quantitative polymerase chain reaction $(R T-q P C R)$. Total RNA was extracted from xenograft tissues using TRIzol reagent (Invitrogen; Thermo Fisher Scientific, Inc.). The quality and quantity of RNA were determined by measuring the absorbance at wavelengths of 260 and $280 \mathrm{~nm}$ using Nano Drop-2000 ultra microspectrophotometer (Thermo Fisher Scientific, Inc.). A total of one $\mu \mathrm{g}$ of RNA was reverse transcribed into cDNA using RevertAidTM first strand cDNA Synthesis kit according to the manufacturer's protocol. C-myc, Cyclin D1 and Axin2 cDNAs were amplified using SYBR Premix Ex Taq kit (Takara Bio, Inc.) with LightCycler 480 PCR system (Roche Diagnostics, Indianopolis, IN, USA). The thermocycling conditions for the PCR assay cycles were as follows: $95^{\circ} \mathrm{C}$ for $5 \mathrm{~min}, 40$ cycles of $95^{\circ} \mathrm{C}$ for $10 \mathrm{sec}, 95^{\circ} \mathrm{C}$ for $10 \mathrm{sec}$ and $60^{\circ} \mathrm{C}$ for $30 \mathrm{sec}$. The mRNA levels of the target gene were normalized to the level of $\beta$-actin using the $2^{-\Delta \Delta \mathrm{Cq}}$ method (14) and were represented as fold induction. The primers used for RT-qPCR were as follows: Forward, 5'-TGA GGAAACGACGAGAACAG-3' and reverse, 5'-ACGAGA GATTCCAGCTCCTC-3' for c-myc; forward, 5'-ATCTCC CTTGATTCAAACGC-3' and reverse, 5'-GCCCAATGA AAGACCAATCT-3' for cyclin D1; forward, 5'-CAGTGAGCT GGTTGTCACCT-3' and reverse, 5'-CTGAGCTGCTCCTTG AAGTG-3' for Axin2; forward, 5'-CCTCTATGCCAACAC AGTGC-3' and reverse, 5'-GTACTCCTGCTTGCTGATCC-3' for $\beta$-actin.

Western blotting. Proteins were extracted from xenograft tissues using radioimmunoprecipitation assay buffer (Sigma-Aldrich; Merck KGaA, Darmstadt, Germany). The concentration of protein was measured using a bicinchoninic acid protein assay kit (Beyotime Institute of Biotechnology, Haimen, China). A total of $50 \mu \mathrm{g}$ protein was subjected to $10 \%$ SDS-polyacrylamide gel and then transferred onto a polyvinylidene difluoride membrane (EMD Millipore, Billerica, MA, USA). Subsequently, the membrane was blocked with $5 \%$ bovine serum albumin (Sigma-Aldrich; Merck KGaA) in Tris-buffered saline with $0.05 \%$ Tween-20 (TBST) for $1 \mathrm{~h}$ at room temperature and then incubated overnight at $4^{\circ} \mathrm{C}$ with the primary antibodies against $\beta$-actin $(1: 1,000)$, Wnt1 (1:500), Wnt2 (1:500), Wnt5a (1:1,000), Dvl-1 (1:500), GSK3 $\beta$ $(1: 1,000)$, p-GSK3 $\beta$ (1:500) or SFRP-2 (1:1,000). Following washing with TBST three times, the membrane was probed with peroxidase conjugated affinity purified goat anti-mouse immunoglobulin G (Origene Technologies, Inc., Beijing, China, 1:1,000 dilution, cat. no. TA100015) at $37^{\circ} \mathrm{C}$ for $2 \mathrm{~h}$. The level of $\beta$-actin was examined as an internal control. The membranes were developed with an ECL kit (Pierce; Thermo Fisher Scientific Inc.), and the gray value of the bands of interest was analyzed by FlourChem V2.0 (ProteinSimple, San Jose, CA, USA).

Immunohistochemistry. Tumor xenografts were fixed immediately following removal, in a $10 \%$ buffered formal in solution for a maximum of $48 \mathrm{~h}$ at room temperature, prior to being dehydrated and paraffin-embedded under vacuum conditions. Slides with paraffin sections (3-5 $\mu \mathrm{m})$ were deparaffinized. Endogenous peroxidase activity was blocked by incubation in $3 \% \mathrm{H}_{2} \mathrm{O}_{2}$ for $10 \mathrm{~min}$ at room temperature. The slides were incubated with the primary anti-CD44v6 antibody (1:200 dilution) or anti-Survivin antibody (1:500 dilution) overnight at $4^{\circ} \mathrm{C}$. Sections were washed with PBS and incubated with biotinylated anti-rabbit secondary antibody for $2 \mathrm{~h}$ at $37^{\circ} \mathrm{C}$. Diaminobenzidine (DAB) $-\mathrm{H}_{2} \mathrm{O}_{2}$ was used as the chromogen to reveal antibody binding sites. A total of five high-powered fields were randomly chosen using an inverted microscope. Positive cells were marked in brown or dark nankeen. The average optical density was quantified with JEDR801D software (Jetta Technology, Nanjing, Jiangsu, China). The staining results were evaluated and scored independently by two pathologists.

Statistical analysis. All experiments were performed at least three times using independent samples. All data are expressed as the mean \pm standard deviation. Statistical analysis was conducted using SPSS software, version 12.0 software (SPSS, Inc., Chicago, IL, USA). The Student's t-test was used to compare the difference between two individual groups, and one-way analysis of variance followed by Dunnett's post hoc testwas used to compare the difference among multiple groups. $\mathrm{P}<0.05$ was considered to indicate a statistically significant difference.

\section{Results}

QYSL decoction inhibits tumor growth. To explore the role of the QYSL decoction on lung cancer, the present studyestablished a C57BL/6 mouse xenograft model using LLC and administered different doses of QYSL decoction to treat mice. In Table I, there were no significant differences among C, LQ and MQ groups $(\mathrm{P}>0.05)$. However, the tumor size in $\mathrm{HQ}$, DDP or HQ+DDP groups was decreased compared with the $\mathrm{C}$ group $(\mathrm{P}<0.05 ; \mathrm{P}<0.01 ; \mathrm{P}<0.01)$. No significant difference was observed between DDP and HQ+DDP groups, however the tumor masses in the DDP and HQ+DDP groups were lower compared with HQ group $(\mathrm{P}<0.01)$. Following this, the tumor inhibition ratio from different groups was analyzed. Compared with $\mathrm{C}$ group, the tumor inhibition ratios of LQ, MQ, HQ, DDP and HQ+DDP groups were increased $(\mathrm{P}<0.01)$. These data demonstrated that high doses of QYSL decoction and DDP significantly suppressed tumor growth, suggesting that QYSL decoction may function as a lung cancer therapeutic.

QYSL decoction inhibits mRNA levels of c-myc, cyclin D1 and Axin 2, detected via RT-qPCR. To examine the mechanism by which QYSL decoction exhibits its inhibitory effects in lung tumor development, the present study first detected the mRNA levels of c-myc, cyclin D1 and Axin 2 following different treatments. In Fig. 1, there was no significant difference between C and LQ groups in the mRNA levels of c-myc, cyclin D1 and Axin2 ( $>>0.05)$. Compared with $\mathrm{C}$ group, the levels of c-myc, cyclin D1 and Axin2 in MQ, HQ, DDP and $\mathrm{HQ}+\mathrm{DDP}$ groups were significantly repressed $(\mathrm{P}<0.01)$. No significant differences in the expression of c-myc among 
Table I. Tumor mass and inhibition ratio of different groups.

\begin{tabular}{llcc}
\hline Groups & $\mathrm{n}$ & Mass $(\mathrm{g})$ & Ratio $(\%)$ \\
\hline C & 8 & $5.12 \pm 0.63$ & 0 \\
LQ & 8 & $4.53 \pm 1.34$ & 13.51 \\
MQ & 8 & $4.27 \pm 0.62$ & 18.32 \\
HQ & 8 & $3.45 \pm 1.05^{\mathrm{a}}$ & 33.86 \\
DDP & 8 & $1.49 \pm 0.68^{\mathrm{b}}$ & 73.01 \\
HQ+DDP & 8 & $1.48 \pm 0.71^{\mathrm{b}}$ & 73.23 \\
\hline
\end{tabular}

Data are expressed as the mean \pm standard deviation. ${ }^{\mathrm{a}} \mathrm{P}<0.05$, ${ }^{\mathrm{b}} \mathrm{P}<0.01$ vs. C, group. C, control group; QYSL, Qiyusanlong; LQ, low dosage of QYSL decoction group; MQ, middle dosage of QYSL decoction group; HQ, high dosage of QYSL decoction group; DDP, Chemotherapy group; HQ+DDP, co-treatment of HQ and DDP.

HQ, DDP and HQ+DDP groups were observed. However, compared with HQ group, the level of cyclin D1 in DDP group were suppressed, however the expression of Axin2 was upregulated $(\mathrm{P}<0.01)$. These data demonstrated that the QYSL decoction and DDP inhibited the expression of c-myc, cyclin D1 and Axin2, however, the QYSL decoction and DDP demonstrated different degrees of inhibition, and the application of QYSL decoction affected the role of DDP to a certain degree.

QYSL decoction exhibits varied effects on Wnt/ $\beta$-catenin pathway-associated proteins, detected via western blotting. To further determine the mechanism of QYSL decoction functioning in tumor inhibition, the protein levels of regulatory proteins in Wnt/ $\beta$-catenin pathway were detected. In Fig. 2A, compared with $\mathrm{C}$ group, different dosages of QYSL decoction and DDP displayed different degrees of inhibition on the protein levels of Wnt1, Wnt2, Wnt5a, GSK3 $\beta$, p-GSK3 $\beta, \beta$-catenin and Dvl-1. Compared with $\mathrm{C}$ group, the protein levels of Wnt1, Wnt2, Wnt5a, GSK3 $\beta$, p-GSK3 $\beta$, $\beta$-catenin and Dvl-1 in LQ, MQ, HQ, DDP and HQ+DDP groups were significantly suppressed (Fig. 2B-H; $\mathrm{P}<0.01$ ), and the HQ+DDP group demonstrated the most significant inhibitory effect on the expression of the aforementioned genes, except $\beta$-catenin. However, compared with the above genes, different doses of QYSL decoction and DDP resulted in different effect on the protein level of SFRP-2. Compared with $\mathrm{C}$ group, the levels of SFRP-2 in LQ, MQ, DDP and HQ+DDP groups were upregulated $(\mathrm{P}<0.01)$, and HQ markedly enhanced the expression of SFRP-2 (Fig. 2I; P<0.01). Overall, the results revealed that the QYSL decoction and DDP modulated the regulatory protein expression levels in $\mathrm{Wnt} / \beta$-catenin pathway.

QYSL decoction inhibits protein signals of CD44V6 and Survivin, detected via immunohistochemistry. CD44 variant isoforms, particularly CD44v6, have been identified as protein markers for metastatic behavior innumerous malignant tumors (15). Elevated expression of Survivin correlates with poor prognosis, tumor recurrence and drug resistance in various human cancers, including
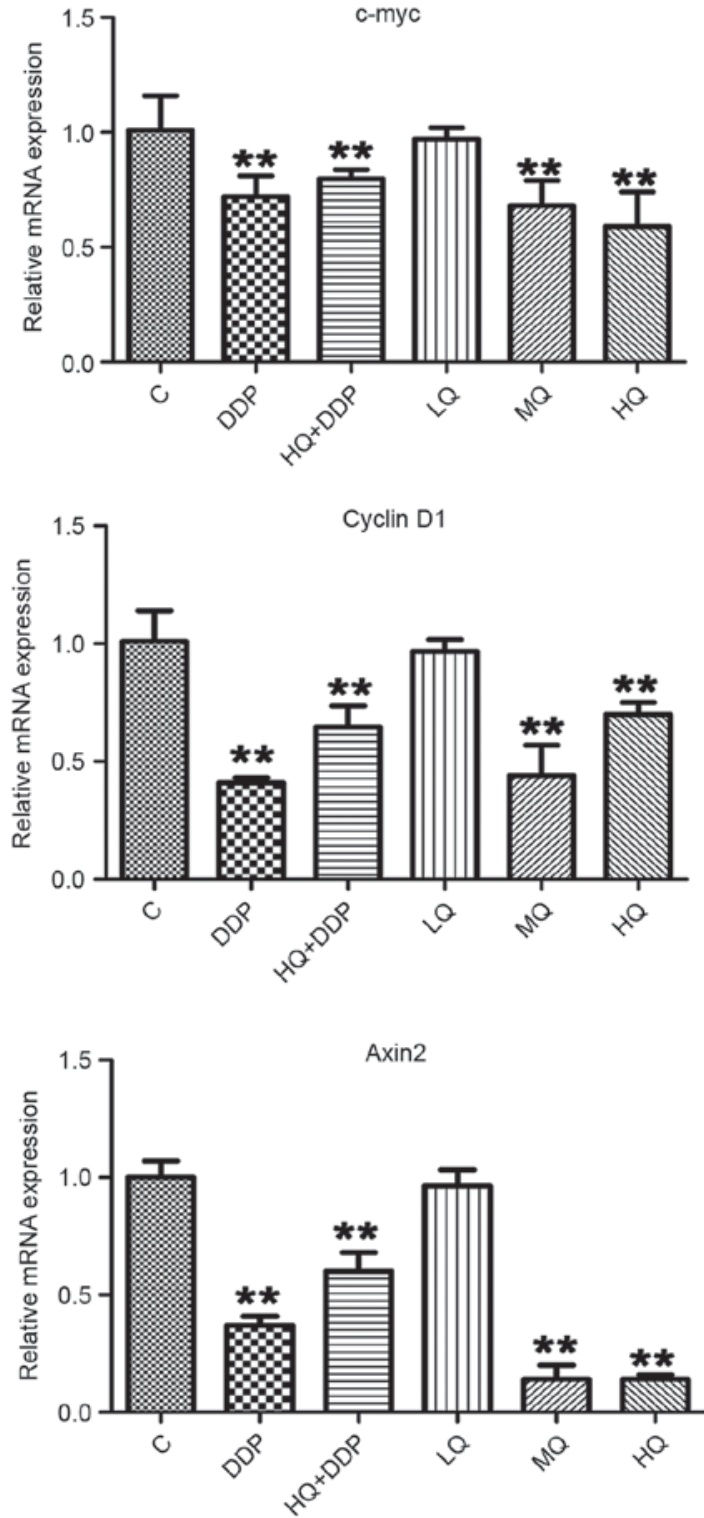

Figure 1. QYSL decoction and DDP inhibit mRNA levels of c-myc, cyclin D1 and Axin2. The established growing xenograft lung cancer mice were randomized into six groups ( $n=8$ per group). i) control group (C): Mice received dosage of $0.2 \mathrm{ml} / 10 \mathrm{~g}$ physiological saline via intragastric administration for 21 days, and $0.4 \mathrm{ml}$ of physiological saline by intraperitoneal injection once a week. ii) Low dosage of QYSL decoction group (LQ): Mice underwent intragastric administration of $20.12 \mathrm{~g} / \mathrm{kg}$ QYSL decoction for 21 days, and $0.4 \mathrm{ml}$ physiological saline via intraperitoneal injection once a week. iii) Medium dosage of QYSL decoction group (MQ): Mice underwent intragastric administration of $40.24 \mathrm{~g} / \mathrm{kg}$ QYSL for 21 days, and $0.4 \mathrm{ml}$ physiological saline via intraperitoneal injection once a week. iv) High dosage of QYSL decoction group (HQ): Mice received intragastric administration with doses of $80.48 \mathrm{~g} / \mathrm{kg}$ QYSL for 21 days, and $0.4 \mathrm{ml}$ physiological saline via intraperitoneal injection once a week. v) DDP group (DDP): Mice received intragastric administration of $0.2 \mathrm{ml} / 10 \mathrm{~g}$ physiological saline for 30 days, and $0.4 \mathrm{ml}$ DDP via intraperitoneal injection once a week. vi) HQ+DDP group: Mice received intragastric administration of $80.48 \mathrm{~g} / \mathrm{kg}$ QYSL for 21 days, and $0.4 \mathrm{ml}$ DDP via intraperitoneal injection once a week. Tumor tissues were isolated for reverse transcription-quantitative polymerase chain reaction. $\beta$-actin was used as the internal control with the quantification method $2^{-\Delta \Delta C q}{ }^{* * *} \mathrm{P}<0.01$ vs. C. DDP, Cisplatin (DDP)-based chemotherapy; QYSL, Qiyusanlong.

lung cancer (16). The present study detected the signals of CD44v6 (Fig. 3) and Survivin (Fig. 4) via immunohistochemistry following different treatments, presented 


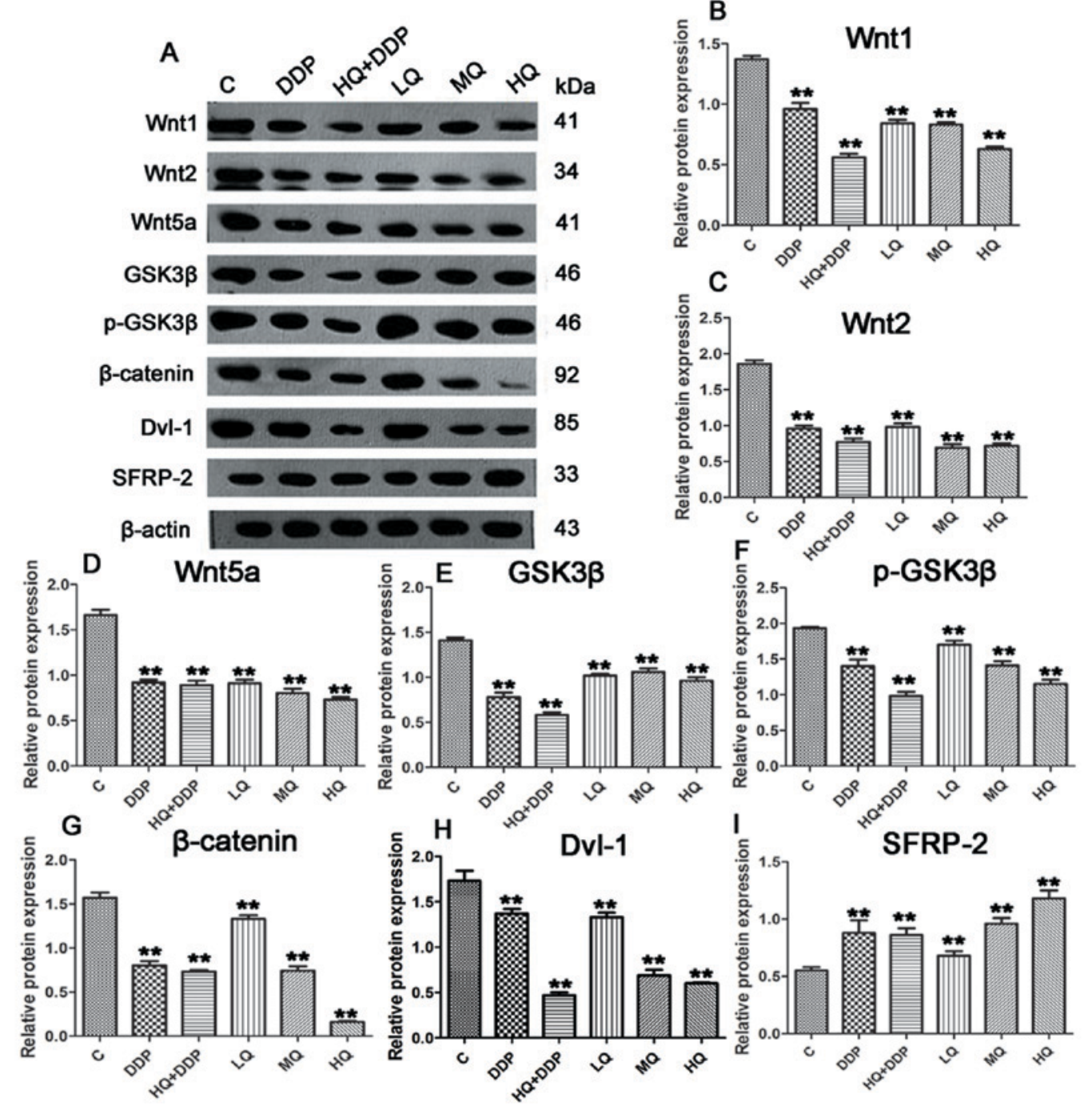

Figure 2. QYSL decoction and DDP modulate the expression levels of regulatory proteins in Wnt/ $\beta$-catenin pathway. The established growing xenograft lung cancer mice were randomized into six groups ( $\mathrm{n}=8$ per group). i) control group (C): Mice received dosage of $0.2 \mathrm{ml} / 10 \mathrm{~g}$ physiological saline via intragastric administration for 21 days, and $0.4 \mathrm{ml}$ of physiological saline by intraperitoneal injection once a week. ii) Low dosage of QYSL decoction group (LQ): Mice underwent intragastric administration of $20.12 \mathrm{~g} / \mathrm{kg}$ QYSL decoction for 21 days, and $0.4 \mathrm{ml}$ physiological saline via intraperitoneal injection once a week. iii) Medium dosage of QYSL decoction group (MQ): Mice underwent intragastric administration of $40.24 \mathrm{~g} / \mathrm{kg}$ QYSL for 21 days, and $0.4 \mathrm{ml}$ physiological saline via intraperitoneal injection once a week. iv) High dosage of QYSL decoction group (HQ): Mice received intragastric administration with doses of $80.48 \mathrm{~g} / \mathrm{kg}$ QYSL for 21 days, and $0.4 \mathrm{ml}$ physiological saline via intraperitoneal injection once a week. v) DDP group (DDP): Mice received intragastric administration of $0.2 \mathrm{ml} / 10 \mathrm{~g}$ physiological saline for 30 days, and $0.4 \mathrm{ml}$ DDP via intraperitoneal injection once a week. vi) HQ+DDP group: Mice received intragastric administration of $80.48 \mathrm{~g} / \mathrm{kg}$ QYSL for 21 days, and $0.4 \mathrm{ml}$ DDP via intraperitoneal injection once a week. Tumor tissues proteins were extracted for western blotting. (A) Representative image and quantification of (B) Wnt1, (C) Wnt 2, (D) Wnt5a, (E) GSK3 $\beta$, (F) p-GSK3 $\beta$, (G) $\beta$-catenin, (H) Dvl-1 and (I) SFRP-2 protein expression levels. The gray value of the bands of interest was normalized to $\beta$-actin and analyzed by FlourChem V2.0. ${ }^{* *} \mathrm{P}<0.01$ vs. (C). DDP, Cisplatin (DDP)-based chemotherapy; QYSL, Qiyusanlong; p, phosphorylataed; GSK3 $\beta$, glycogen synthase kinase 3 $\beta$; Dvl, Dishevelled; SFRP, Secreted frizzled-related proteins.

in Table II. In Fig. 3, the signals of CD44v6 in MQ, HQ, DDP and HQ+DDP groups decreased compared with C group $(\mathrm{P}<0.05)$, and there were no significant differences among MQ, HQ and DDP groups, however HQ+DDP group revealed the most significant inhibitory effect. Similarly, there was no significant differences observed in Survivin signals among $\mathrm{C}$ and LQ groups $(\mathrm{P}>0.05)$. However, the signals of Survivin in MQ, HQ, DDP and HQ+DDP groups were suppressed compared with the $\mathrm{C}$ group (Fig. 4; $\mathrm{P}<0.05)$, and there was no significant difference among the MQ, HQ, DDP and HQ+DDP groups ( $>0.05)$. These data revealed that QYSL decoction and DDP inhibited the protein levels of CD44v6 and Survivin.

\section{Discussion}

Lung cancer is a leading contributor to cancer-associated mortalities worldwide (4). Chemotherapy has improved overall survival, however remains limited at $<12$ months median overall survival (17). Adverse effects, including nausea and vomiting, sore mouth, diarrhea, hepatotoxicity and immunosuppression, are commonly reportedin patients with cancers treated with chemotherapy. A previous report suggested that a variety of TCM may extensively be used for managing these adverse effects (18). QYSL decoction, established by Professor Han Mingxiang, an old doctor of TCM according to the folk prescription of 'Fu Zheng Xiao Ji', was composed from 10 
Table II. Signals of CD44v6 and Survivin in tumor tissues from different groups.

\begin{tabular}{lccc}
\hline Groups & $\mathrm{n}$ & CD44v6 & Survivin \\
\hline C & 6 & $0.85 \pm 0.12$ & $0.83 \pm 0.10$ \\
LQ & 6 & $0.81 \pm 0.07$ & $0.83 \pm 0.11$ \\
MQ & 6 & $0.75 \pm 0.06^{\mathrm{a}}$ & $0.76 \pm 0.05^{\mathrm{a}}$ \\
HQ & 6 & $0.74 \pm 0.08^{\mathrm{a}}$ & $0.71 \pm 0.07^{\mathrm{a}}$ \\
DDP & 6 & $0.74 \pm 0.08^{\mathrm{a}}$ & $0.72 \pm 0.07^{\mathrm{a}}$ \\
HQ+DDP & 6 & $0.65 \pm 0.11^{\mathrm{a}}$ & $0.71 \pm 0.11^{\mathrm{a}}$ \\
\hline
\end{tabular}

Data are expressed as the mean \pm standard deviation. ${ }^{\mathrm{a}} \mathrm{P}<0.05$ vs. $\mathrm{C}$, group. C, control group; QYSL, Qiyusanlong; LQ, low dosage of QYSL decoction group; MQ, middle dosage of QYSL decoction group; $\mathrm{HQ}$, high dosage of QYSL decoction group; DDP, Chemotherapy group; HQ+DDP, co-treatment of HQ and DDP; CD44v6, cluster of differentiation 44 variation 6 .
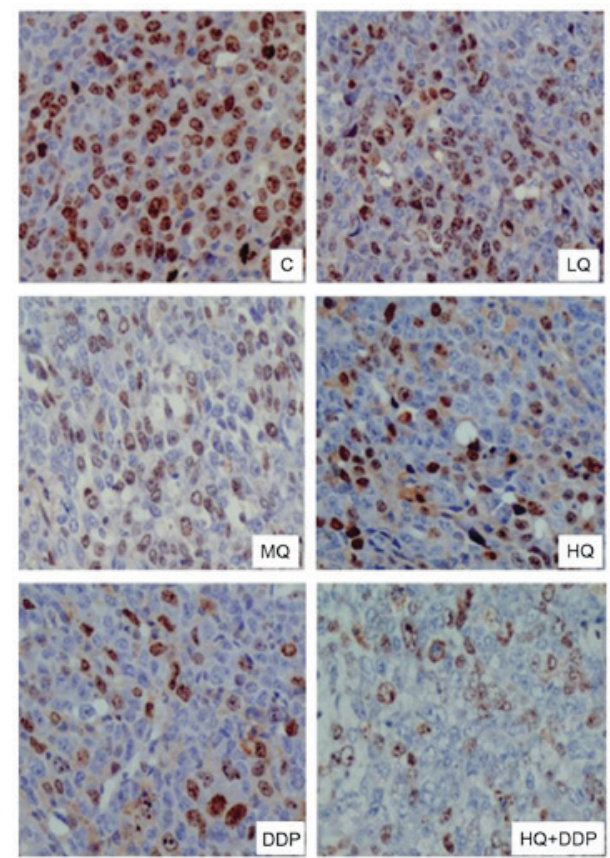

Figure 3. QYSL decoction and DDP repress the signals ofcluster of differentiation 44 variation 6 . The established growing xenograft lung cancer mice were randomized into six groups ( $n=8$ per group). i) control group (C): Mice received dosage of $0.2 \mathrm{ml} / 10 \mathrm{~g}$ physiological saline via intragastric administration for 21 days, and $0.4 \mathrm{ml}$ of physiological saline by intraperitoneal injection once a week. ii) Low dosage of QYSL decoction group (LQ): Mice underwent intragastric administration of $20.12 \mathrm{~g} / \mathrm{kg}$ QYSL decoction for 21 days, and $0.4 \mathrm{ml}$ physiological saline via intraperitoneal injection once a week. iii) Medium dosage of QYSL decoction group (MQ): Mice underwent intragastric administration of $40.24 \mathrm{~g} / \mathrm{kg}$ QYSL for 21 days, and $0.4 \mathrm{ml}$ physiological saline via intraperitoneal injection once a week. iv) High dosage of QYSL decoction group (HQ): Mice received intragastric administration with doses of $80.48 \mathrm{~g} / \mathrm{kg}$ QYSL for 21 days, and $0.4 \mathrm{ml}$ physiological saline via intraperitoneal injection once a week. v) DDP group (DDP): Mice received intragastric administration of $0.2 \mathrm{ml} / 10 \mathrm{~g}$ physiological saline for 30 days, and $0.4 \mathrm{ml}$ DDP via intraperitoneal injection once a week. vi) HQ+DDP group: Mice received intragastric administration of $80.48 \mathrm{~g} / \mathrm{kg}$ QYSL for 21 days, and $0.4 \mathrm{ml} \mathrm{DDP}$ via intraperitoneal injection once a week. The tumor tissues were extracted for immunohistochemistry. A total offive high-powered fields were randomly selected using an inverted microscope. Positive cells were marked in brown or dark nankeen. The average optical density was quantified with JEDR801D software. DDP, Cisplatin (DDP)-based chemotherapy; QYSL, Qiyusanlong.
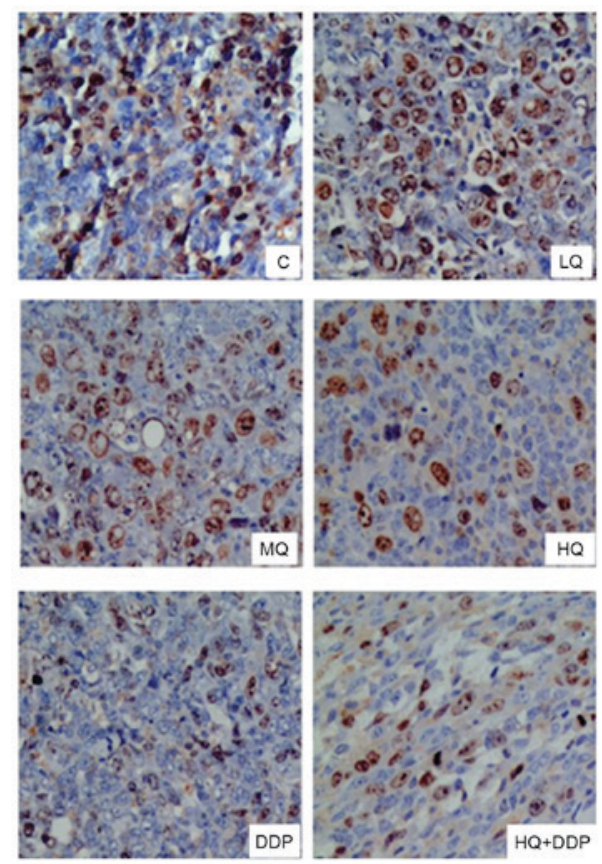

Figure 4. QYSL decoction and DDP repress the signals of Survivin. The established growing xenograft lung cancer mice were randomized into six groups ( $\mathrm{n}=8$ per group). i) control group (C): Mice received dosage of $0.2 \mathrm{ml} / 10 \mathrm{~g}$ physiological saline via intragastric administration for 21 days, and $0.4 \mathrm{ml}$ of physiological saline by intraperitoneal injection once a week. ii) Low dosage of QYSL decoction group (LQ): Mice underwent intragastric administration of $20.12 \mathrm{~g} / \mathrm{kg}$ QYSL decoction for 21 days, and $0.4 \mathrm{ml}$ physiological saline via intraperitoneal injection once a week. iii) Medium dosage of QYSL decoction group (MQ): Mice underwent intragastric administration of $40.24 \mathrm{~g} / \mathrm{kg}$ QYSL for 21 days, and $0.4 \mathrm{ml}$ physiological saline via intraperitoneal injection once a week. iv) High dosage of QYSL decoction group (HQ): Mice received intragastric administration with doses of $80.48 \mathrm{~g} / \mathrm{kg}$ QYSL for 21 days, and $0.4 \mathrm{ml}$ physiological saline via intraperitoneal injection once a week. v) DDP group (DDP): Mice received intragastric administration of $0.2 \mathrm{ml} / 10 \mathrm{~g}$ physiological saline for 30 days, and $0.4 \mathrm{ml}$ DDP via intraperitoneal injection once a week. vi) HQ+DDP group: Mice received intragastric administration of $80.48 \mathrm{~g} / \mathrm{kg}$ QYSL for 21 days, and $0.4 \mathrm{ml}$ DDP via intraperitoneal injection once a week. The tumor tissues were extracted for immunohistochemistry. A total offive high-powered fields were randomly selected using an inverted microscope. Positive cells were marked with brown or dark nankeen. The average optical density was quantified with JEDR801D software. DDP, Cisplatin (DDP)-based chemotherapy; QYSL, Qiyusanlong.

kinds of Chinese medicine including astragalus membranaceus (Huangqi), polygonatum odoratum (yuzu), scolopendra (tianlong), pberetima (dilong), solanum nigrum (longkui), herbahedyotis (baihushecao), semen coicis (yiyiren), euphorbia helioscopia (zeqi), curcuma longa (eshu) and tendril-leaved fritillary bulb (chuanbei). The function of QYSL decoction in cancer therapy has not been fully elucidated. The present study aimed to expand current treatments of lung cancer and provide a novel candidate as alung cancer treatment. The present study employed the QYSL decoction to treat lung cancer xenografts in nude mice models to observe tumor growth following different treatments. The results revealed that QYSL decoction regulated tumor growth via modulating the regulatory proteins in the Wnt/ $/$-catenin pathway.

QYSL decoction was composed of 10 types of Chinese medicine. These aforementioned herbs have previously been categorized to exhibit anti-cancer properties. Astragalus membranaceus (Huangqi) has a wide range of 
immunopotentiating effects and is widely used as an adjuvant medicine during cancer therapy (19). Polygonatum odoratum (yuzu) extracts induce apoptosis of MDA-MB-231 breast cancer cells (20) and A549 human lung cancer cells (21). Extracts of scolopendra (tianlong) subspinipes mutilans induce cell cycle arrest and apoptosis in A375 human melanoma cells (22). Aqueous extract of solanum nigrum (longkui) leaves suppress tumor growth and enhance cytotoxicity of DDP (23). HerbaHedyotidis (baihushecao) is the 'assistant and attendant' herb with 'bitter and cold' properties, resulting in 'heat-clearing and detoxification' and 'pain-alleviating' effects to reduce or control tumor size (7). The components of semen coicis(yiyiren) exert anti-cancer actions via inhibiting proliferation and inducing apoptosis of cancer cells, in addition to increasing the sensitivity of patients to chemotherapeutic agents (24). Euphorbia helioscopia (zeqi) extract inhibits hepatocellular carcinoma growth in nude mice xenografts (25). A previous study indicated that curcuma longa (eshu) exerts anti-cancer effects in a variety of biological pathways involved in mutagenesis, apoptosis, tumorigenesis, cell cycle regulation and metastasis (26). These previous reports indicate that the components of QYSL decoction exhibit anti-tumor functions in various cancers. The present study demonstrated that the application of QYSL decoction suppressed tumor growth, which was in agreement with previous reports. These data provided evidence that QYSL decoction maybe used as a novel therapy for lung cancer treatment.

Wnt/ $\beta$-catenin signaling is an evolutionarily conserved and versatile pathway that is known to be involved in embryonic development, tissue homeostasis and a wide variety of human diseases. In the present study, the administration of QYSL decoction and DDP affected the mRNA and protein levels of the regulatory proteins in the $\mathrm{Wnt} / \beta$-catenin pathway, suggesting that QYSL decoction may interfere with lung cancer development via the Wnt/ $\beta$-catenin pathway. Stimulation of the canonical Wnt pathway ultimately results in the activation of $\beta$-catenin, which promotes the transcription of proteins involved in cell proliferation, including c-Myc and Cyclin D1 (27). The present study demonstrated that the application of a high dose of QYSL decoction significantly reduced the expression levels of c-myc, Cyclin D1, Axin2, Wnt1, Wnt2, Wnt5a, GSK $3 \beta, \mathrm{p}$-GSK $3 \beta$ and $\beta$-catenin, suggesting that QYSL decoction interacts with the Wnt/ $\beta$-catenin pathway. Dvl family proteins located upstream of the Wnt pathway are overexpressed in lung cancer (28). Dvl-1 and Dvl-3 affected the biological behavior of lung cancer cells via canonical and non canonical Wnt pathway (28). In the present study, QYSL decoction, mimicking chemotherapy DDP, suppressed the expression of Dvl-1 and suppressed the cyclin D1, c-myc and axin 2 levels. These results revealed that there may be a new mechanism by which Dvl-1 was associated with lung cancer development. Therefore, further investigations need to be performed in our further work. SFRP2, an identified member of the SFRPs family of molecules, is able to inhibit the Wnt-induced increase of free $\beta$-catenin and influence cell cycle progression and tumor cell proliferation (29). CD44v6, a downstream factor of Wnt signaling, acts as a marker for predicting cancer metastasis (30). The knockdown of CD44v6 expression may result in depression of tumor metastases and cell invasion (31). Survivin, a member of an apoptosis inhibitor family, was a unique target for tumor therapy (32). DDP and QYSL decoction repressed the signals of CD44v6 and survivin. Notably, the effect of QYSL decoction was milder compared with DDP, suggesting that TCM QYSL decoction may be more effectively tolerated than DDP.

In conclusion, the results of the present study demonstrated that different doses of QYSL decoction resulted in various effects on tumor growth and the expression of regulatory proteins in the $\mathrm{Wnt} / \beta$-catenin pathway. The combination of DDP and a high dose of QYSL decoction significantly enhanced the inhibitory effect on lung cancer. Therefore, clinical trials may attempt to administer high doses of QYSL decoction combined with DDP in cancer therapy in the future. However, the effective doses of QYSL decoction and DDP on cancer inhibition require further investigation. QYSL decoction and DDP repressed lung cancer development via monitoring the expression of regulatory proteins in Wnt/ $\beta$-catenin pathway. Furthermore, the effect of QYSL decoction was milder, however when combined with the chemotherapy drug, enhanced the overall inhibitory effect. Further investigations may help provide novel strategies for lung cancer therapy by targeting the Wnt signaling pathway in the future.

\section{Acknowledgements}

The present study was supported by the Natural Science Foundation of Anhui Province (grant no. 1708085MH197).

\section{References}

1. Torre LA, Bray F, Siegel RL, Ferlay J, Lortet-Tieulent J and Jemal A: Global cancer statistics, 2012. CA Cancer J Clin 65: 87-108, 2015.

2. Hwang KE and Kim HR: Response evaluation of chemotherapy for lung cancer. Tuberc Respir Dis (Seoul) 80: 136-142, 2017.

3. Yu N, Xiong Y and Wang C: Bu-Zhong-Yi-Qi decoction, the water extract of chinese traditional herbal medicine, enhances cisplatin cytotoxicity in A549/DDP cells through induction of apoptosis and autophagy. Biomed Res Int 2017: 3692797, 2017.

4. Somasundaram A and Burns TF: The next generation of immunotherapy: Keeping lung cancer in check. J Hematol Oncol 10: 87, 2017.

5. Ou Y, Zhai D, Wu N and Li X: Downregulation of miR-363 increases drug resistance in cisplatin-treated HepG2 by dysregulating Mcl-1. Gene 572: 116-122, 2015.

6. Lin HQ, Gong AG, Wang HY, Duan R, Dong TT, Zhao KJ and Tsim KW: Danggui Buxue Tang (Astragali Radix and Angelicae Sinensis Radix) for menopausal symptoms: A review. J Ethnopharmacol 199: 205-210, 2017.

7. Liu Z, Chen S, Cai J, Zhang E, Lan L, Zheng J, Liao L, Yang X, Zhou C and Du J: Traditional Chinese medicine syndrome-related herbal prescriptions in treatment of malignant tumors. J Tradit Chin Med 33: 19-26, 2013.

8. Zhang X, Tong J and Li Z: Qiyusanlong decoction inhibits the level of PD-1/PD-L1 in mice bearing Lewis lung carcinoma. Xi Bao Yu Fen Zi Mian Yi Xue Za Zhi 32: 770-774, 2016 (In Chinese).

9. Jiang Q, He M, Guan S, Ma M, Wu H, Yu Z, Jiang L, Wang Y, Zong X, Jin F and Wei M: MicroRNA-100 suppresses the migration and invasion of breast cancer cells by targeting FZD-8 and inhibiting Wnt $/ \beta$-catenin signaling pathway. Tumour Biol 37: 5001-5011, 2016.

10. Zhan P, Zhang B, Xi GM, Wu Y, Liu HB, Liu YF, Xu WJ, Zhu QQ, Cai F, Zhou ZJ, et al: PRC1 contributes to tumorigenesis of lung adenocarcinoma in association with the $\mathrm{Wnt} / \beta$-catenin signaling pathway. Mol Cancer 16: 108, 2017.

11. Hu T and Li C: Convergence between Wnt- $\beta$-catenin and EGFR signaling in cancer. Mol Cancer 9: 236, 2010. 
12. Shang $\mathrm{S}$, Hua $\mathrm{F}$ and $\mathrm{Hu} \mathrm{ZW}$ : The regulation of $\beta$-catenin activity and function in cancer: Therapeutic opportunities. Oncotarget 8: 33972-33989, 2017.

13. Salahshor S, Naidoo R, Serra S, Shih W, Tsao MS, Chetty R and Woodgett JR: Frequent accumulation of nuclear E-cadherin and alterations in the Wnt signaling pathway in esophageal squamous cell carcinomas. Mod Pathol 21: 271-281, 2008.

14. Livak KJ and Schmittgen TD: Analysis of relative gene expression data using real-time quantitative PCR and the 2(-Delta Delta C(T)) method. Methods 25: 402-408, 2001.

15. Ponta H, Sherman L and Herrlich PA: CD44: From adhesion molecules to signalling regulators. Nat Rev Mol Cell Biol 4: 33-45, 2003.

16. Wang S, Zhu L, Zuo W, Zeng Z, Huang L, Lin F, Lin R, Wang J, Lu J, Wang Q, et al: MicroRNA-mediated epigenetic targeting of survivin significantly enhances the antitumor activity of paclitaxel against non-small cell lung cancer. Oncotarget 7: 37693-37713, 2016.

17. Zhuang B, Du L, Xu H, Xu X, Wang C, Fan Y, Cong M, Yin J, Li H and Guan H: Self-assembled Micelle Loading Cabazitaxel for therapy of Lung Cancer. Int J Pharm 499: $146-155,2016$

18. Taixiang W, Munro AJ and Guanjian L: Chinese medical herbs for chemotherapy side effects in colorectal cancer patients. Cochrane Database Syst Rev: CD004540, 2005

19. Xiao WL, Motley TJ, Unachukwu UJ, Lau CB, Jiang B Hong F, Leung PC, Wang QF, Livingston PO, Cassileth BR and Kennelly EJ: Chemical and genetic assessment of variability in commercial Radix Astragali (Astragalus spp.) by ion trap LC-MS and nuclear ribosomal DNA barcoding sequence analyses. J Agric Food Chem 59: 1548-1556, 2011.

20. Tai Y, Sun YM, Zou X, Pan Q, Lan YD, Huo Q, Zhu JW, Guo F, Zheng CQ, Wu CZ and Liu H: Effect of Polygonatum odoratum extract on human breast cancer MDA-MB-231 cell proliferation and apoptosis. Exp Ther Med 12: 2681-2687, 2016.

21. Wu L, Liu T, Xiao Y, Li X, Zhu Y, Zhao Y, Bao J and Wu C: Polygonatum odoratum lectin induces apoptosis and autophagy by regulation of microRNA-1290 and microRNA-15a-3p in human lung adenocarcinoma A549 cells. Int J Biol Macromol 85 217-226, 2016.
22. Ma W, Liu R, Qi J and Zhang Y: Extracts of centipede Scolopendra subspinipes mutilans induce cell cycle arrest and apoptosis in A375 human melanoma cells. Oncol Lett 8: 414-420, 2014.

23. Yang MY, Hung $\mathrm{CH}$, Chang $\mathrm{CH}$, Tseng $\mathrm{TH}$ and Wang $\mathrm{CJ}$ Solanum nigrum suppress angiogenesis-mediated tumor growth through inhibition of the AKT/mTOR pathway. Am J Chin Med 44: 1273-1288, 2016.

24. Liu X, Xu F, Wang G, Diao X and Li Y: Kanglaite injection plus chemotherapy versus chemotherapy alone for non-small cell lung cancer patients: A systematic review and meta-analysis. Curr Ther Res Clin Exp 69: 381-411, 2008.

25. Cheng J, Han W, Wang Z, Shao Y, Wang Y, Zhang Y, Li Z, Xu X and Zhang Y: Hepatocellular carcinoma growth is inhibited by euphorbia helioscopia L. Extract in nude mice xenografts. Biomed Res Int 2015: 601015, 2015.

26. Kocaadam B and Sanlier N: Curcumin, an active component of turmeric (Curcuma longa), and its effects on health. Crit Rev Food Sci Nutr 57: 2889-2895, 2017.

27. MacDonald BT, Tamai K and He X: Wnt/beta-catenin signaling: Components, mechanisms, and diseases. Dev Cell 17: 9-26, 2009.

28. Zhao Y, Yang ZQ, Wang Y, Miao Y, Liu Y, Dai SD, Han Y and Wang EH: Dishevelled-1 and dishevelled-3 affect cell invasion mainly through canonical and noncanonical Wnt pathway, respectively and associate with poor prognosis in nonsmall cell lung cancer. Mol Carcinog 49: 760-770, 2010.

29. Liu Y, Zhou Q, Zhou D, Huang C, Meng X and Li J: Secreted frizzled-related protein 2-mediated cancer events: Friend or foe? Pharmacol Rep 69: 403-408, 2017.

30. Wang J, Xiao L, Luo CH, Zhou H, Zeng L, Zhong J, Tang Y, Zhao XH, Zhao $\mathrm{M}$ and Zhang Y: CD44v6 promotes $\beta$-catenin and TGF- $\beta$ expression, inducing aggression in ovarian cancer cells. Mol Med Rep 11: 3505-3510, 2015.

31. Sun W and Chen G: Impact and mechanism of non-steroidal anti-inflammatory drugs combined with chemotherapeutic drugs on human lung cancer-nude mouse transplanted tumors. Oncol Lett 11: 4193-4199, 2016.

32. Garg H, Suri P, Gupta JC, Talwar GP and Dubey S: Survivin: A unique target for tumor therapy. Cancer Cell Int 16: 49, 2016. 